\title{
Looking at European Architecture. A Critical View, Collectif
}

Bruxelles, Éditions CIVA, 2008

Hélène Jannière

\section{(2) OpenEdition}

\section{Journals}

Édition électronique

URL : http://journals.openedition.org/crau/332

DOI : $10.4000 /$ crau.332

ISSN : 2547-5746

\section{Éditeur}

Éditions du patrimoine

Édition imprimée

Date de publication : 1 décembre 2009

Pagination : 218-220

ISBN : 978-2-85822-944-4

ISSN : 1296-4077

\section{Référence électronique}

Hélène Jannière, «Looking at European Architecture. A Critical View, Collectif », Les Cahiers de la

recherche architecturale et urbaine [En ligne], 24/25 | 2009, mis en ligne le 01 septembre 2017, consulté le 24 septembre 2020. URL : http://journals.openedition.org/crau/332 ; DOI : https://doi.org/10.4000/ crau.332

Ce document a été généré automatiquement le 24 septembre 2020

Cahiers de la recherche architecturale et urbaine 


\title{
Looking at European Architecture. A Critical View, Collectif
}

\author{
Bruxelles, Éditions CIVA, 2008
}

Hélène Jannière

\section{RÉFÉRENCE}

Looking at European Architecture. A Critical View, Collectif, Bruxelles, Éditions CIVA, 2008.464 pages, $15 €$.

1 Ce livre est le fruit d'une initiative du réseau européen gau:di (gouvernance, architecture et urbanisme: démocratie et interaction), soutenu par le programme Culture 2000 (Commission européenne) et coordonné par le Centre international pour la Ville, l'Architecture et le Paysage (CIVA, Bruxelles) ${ }^{1}$. En association avec le Royal Institute of British Architects (RIBA), l'ouvrage rassemble des écrits critiques provenant des vingt-sept États membres de l'Union européenne, au terme d'un processus de sélection mené en première étape par des correspondants nationaux, et poursuivi par un jury composé de Jean-Louis Cohen, Françoise Fromonot, Joseph Rykwert et Špela Hudnik. Les deux coordinateurs de l'ouvrage, Irena Murray (British Architectural Library) et Christophe Pourtois (CIVA), affichent pour ce recueil l'ambition « d'effectuer un itinéraire architectural et linguistique à travers une Europe généreuse en diversités, laissant le lecteur rêveur parmi la multiplicité descriptive et conceptuelle offerte par les ressources des langues européennes ${ }^{2} »$. On sera ainsi sensible à la préoccupation - peut-être liée au cadre institutionnel qui est à l'origine de ce livre d'offrir à côté de la traduction anglaise chaque article dans sa langue originale : les deux coordinateurs présentent e choix comme un reflet de leur conception de la critique, qui comporte selon eux une indéniable composante littéraire.

2 On ne peut que saluer cette initiative qui rend accessibles en anglais vingt-sept aperçus de l'état actuel de l'architecture européenne et de ses représentations, iffractées par autant d'écritures critiques, et marquer de l'intérêt pour le processus dont elle est le 
fruit. Ces textes, dont les parutions originales s'échelonnent de 2001 à 2007, n'ont pas été écrits en réponse à un appel à contributions, ce qui aurait pu offrir une image quelque peu artificielle de la critique européenne. La plupart sont extraits de revues professionnelles ou de recueils annuels sur l'architecture; dans quelques cas, ce sont des conférences ou des textes publiés à l'occasion d'expositions, comme «La vie rêvée des Belges", introduction de Maurizio Cohen au pavillon belge de la Biennale d'architecture de Venise en 2002. Au-delà des contenus critiques eux-mêmes, l'un des objectifs de ce recueil semble être de refléter la diversité des médias qui forment les canaux européens de diffusion de l'architecture, depuis des revues établies et internationalement connues à des revues plus jeunes et plus marginales. Cette anthologie montre aussi le rôle important de la presse quotidienne dans une critique engagée et de qualité. Chaque article s'accompagne d'informations sur le média d'où est extrait le texte reproduit: ainsi, pour les revues, cette brève fiche technique livre la date de fondation, le tirage et le nom du rédacteur en chef, ainsi qu'une courte note biographique sur l'auteur de l'article. Ce dernier point permet de constater l'émergence d'une nouvelle génération de critiques nés dans les années 1970, actifs tant dans les médias que dans les institutions d'enseignement.

\section{Looking at European architecture ou Looking at European criticism?}

3 Malgré des objectifs dignes d'intérêt, on peut toutefois regretter l'absence d'une orientation claire pour cet ouvrage dont, in fine, on saisit assez mal l'objet. Celui-ci oscille entre un aperçu de la critique européenne et un panorama des nouvelles conditions et des nouveaux enjeux de l'architecture dans les vingt-sept pays membres. Cette hésitation se révèle particulièrement avec la juxtaposition des quatre courts essais des membres du jury, sur lesquels s'ouvre le recueil. Deux d'entre eux portent sur la critique. Joseph Rykwert ${ }^{3}$ juge utile, par rapport à l'actuelle prolifération de médias, de revenir brièvement aux fonctions premières de la critique en se référant à Vitruve, pour qui l'art de bâtir n'a pu progresser qu'en même temps que l'art de critiquer, et il rappelle que la critique repose nécessairement sur une position théorique, justifiable par une argumentation rationnelle. Dans un tout autre registre, Françoise Fromonot ${ }^{4}$ dresse un tableau pessimiste des conditions actuelles de l'exercice de la critique: «agonie » des revues de tendance, absence dans la presse d'analyses négatives des édifices, autocensure qui s'explique par la dépendance des critiques par rapport aux revues et par la «consanguinité » du «milieu » architectural. Les deux autres textes évoquent les nouvelles conditions du projet architectural et urbain dans une Europe livrée, après 1989, à la généralisation de l'économie de marché et au capitalisme néo-libéral actuellement à la " conquête de l'Est », de ses citoyens et de ses espaces urbains, comme le souligne Špela Hudnik. Dans une synthèse sur la « reconstruction symbolique » des villes européennes après l'ère moderniste, JeanLouis Cohen souligne de son côté la tendance à la monumentalisation de l'architecture, qui va chercher de plus en plus dans le répertoire des arts plastiques les instruments de son expressivité mais aussi de sa propre médiatisation ${ }^{5}$. Cette contribution fait écho à plusieurs autres articles, dont celui de Pippo Ciorra ${ }^{6}$ sur l'« effet Bilbao ", terme lancé par Giorgio Muratore et Sebastian Redecke dans la revue Bauwelt en 1999. 
Parmi les vingt-sept «morceaux choisis », les découvertes les plus intéressantes sont sans nul doute les écrits en provenance des «nouveaux » pays de l'Union européenne. Leur intérêt le plus patent est de proposer une critique architecturale souvent plus acérée et plus engagée. Plusieurs auteurs dressent ainsi un tableau sans concession de la situation de l'architecture dans leur pays: ils regrettent notamment l'absence de «culture architecturale» ou le manque de considération des pouvoirs publics pour l'architecture, pour les valeurs civiques et les valeurs symboliques représentatives de la sphère publique qu'elle pourrait incarner. La plupart des auteurs (des nouveaux pays membres comme ceux d'Europe occidentale) dénoncent le fait que ces valeurs soient de plus en plus fréquemment niées par les impératifs des investisseurs privés, comme le rappelle Sebastian Redecke dans son article sur le centre-ville de Magdeburg, ou qu'elles soient confisquées par les nécessités du «spectacle " - de l'entertainment dominant dans la société du marché généralisé, comme l'analyse Luis FernándezGaliano dans "Spectacle and Its Discontents ; or, the Elusive Joys of Architainment ${ }^{7}$ ".

À la lecture des différentes contributions rassemblées dans l'ouvrage, plusieurs problématiques de l'architecture et de l'urbanisme européens contemporains peuvent in fine être identifiées : parmi celles-ci, la généralisation du sprawl urbain, la relation entre espace public et espace privé, l'espace urbain livré aux intérêts privés, notamment lors de la rénovation des villes de l'ancien bloc de l'Est, ou encore l'événement tenant lieu de projet urbain. D'une manière générale, la question de la relation de l'architecture à l'intérêt public, via la relation de l'architecture aux stratégies d'aménagement urbain, forme l'un des thèmes dominants du recueil.

Même si un certain nombre de thématiques se dégagent a posteriori, on peut cependant regretter que l'ouvrage lui-même nous propose finalement assez peu de recul analytique. Il est vrai que le lecteur est averti, dès l'introduction, des «limites d'un exercice ambitieux et relativement périlleux » qui ne prétend pas "à l'exhaustivité ni même à un caractère anthologique». Ces textes dessinent-ils toutefois les contours d'une « critique européenne » ? Celle-ci est-elle, ainsi que l'espère Françoise Fromonot (qui salue par ailleurs la jeunesse et la virulence de nombreux auteurs), représentative d'un changement de point de vue qui observerait « l'architecture comprise depuis ses effets plutôt qu'à partir de son domaine propre ${ }^{8}$ " ? Il est à souhaiter que cette tentative européenne puisse être poursuivie par un nouveau volume qui tenterait d'adjoindre à la collecte d'écrits, des analyses plus distanciées des articles reproduits, explorant leurs stratégies d'écriture et leurs thèmes dominants, ou encore permettant de réfléchir sur les questions qu'un tel panorama permettrait de faire émerger à propos de l'architecture en Europe.

\section{NOTES}

1. La coordination générale de gau :di est assurée par le Centre international pour la Ville, l'Architecture et le Paysage (CIVA, Bruxelles); ce programme fédère six organisations de diffusion et de conservation de l'architecture (CIVA, Bruxelles; Cité de l'Architecture et du 
Patrimoine, Paris; Fundació Mies van der Rohe, Barcelone; The Architecture Foundation, Londres ; The Lighthouse, Glasgow ; Museum of Finnish Architecture, Helsinki).

2. Irena Murray, Christophe Pourtois, «Introduction », in Looking at European Architecture. A Critical View,Bruxelles, CIVA, 2008, p. 13.

3. Joseph Rykwert, « On Criticism », ibid., pp. 19-20.

4. Françoise Fromonot, « Éloge de l'observation »,ibid., pp. 23-27.

5. Jean-Louis Cohen, « À la recherche du monument perdu »,ibid.,pp. 35-38.

6. Pippo Ciorra, « A Territory without Conflicts »,ibid.,pp. 269-281.

7.

Initialement paru dansHarvard Design Magazine, $\mathrm{n}^{\circ}$

12, Cambridge, Mass., 2000, reproduit pp. 420-425 de l'ouvrage.

8. F. Fromonot, art. cit. note 4, p. 26.

\section{AUTEUR}

\section{HÉLÈNE JANNIÈRE}

Architecte, docteur en histoire de l'art, professeur en histoire et culture architecturale à l'Énsa de Paris-La-Villette. 